\title{
没有 $\boldsymbol{K}_{4}$-图子式的图的邻点可区别全染色
}

\author{
王维凡 (1)*, 王平 (2)
}

(1) 浙江师范大学数学系, 金华 321004

(2) Department of Mathematics, Statistics and Computer Science, St. Francis Xavier University, Antigonish, Nova Scotia B2G2W5, Canada

E-mail: wwf@zjnu.cn; pwang@stfx.ca

收稿日期: 2009-01-12；接受日期: 2009-05-22；＊通信作者

国家自然科学基金 (批准号: 10771197), The James Chair at St. Francis Xavier University 和 Natural Sciences and Engineering Research Council of Canada 资助项目

摘要 图 $G$ 的邻点可区别全染色是 $G$ 的一个正常全染色, 使得每一对相邻顶点有不同 的颜色集合. $G$ 的邻点可区别全色数 $\chi_{a}^{\prime \prime}(G)$ 是使得 $G$ 有一个 $k$-邻点可区别全染色的最小 的整数 $k$. 本文完整刻画了没有 $K_{4}$-图子式的图的邻点可区别全色数. 证明了: 如果 $G$ 是一 个满足最大度 $\Delta \geqslant 3$ 且没有 $K_{4}$-图子式的图, 则 $\Delta+1 \leqslant \chi_{a}^{\prime \prime}(G) \leqslant \Delta+2$, 且 $\chi_{a}^{\prime \prime}(G)=\Delta+2$ 当且仅当 $G$ 中含有两个相邻最大度点.

关键词邻点可区别全染色 没有 $K_{4}$-图子式的图 最大度

$\operatorname{MSC}(2000)$ 主题分类 $05 \mathrm{C} 15$

\section{1 引言}

本文只考虑没有孤立边的简单图. 如果一个图 $H$ 能从另一个图 $G$ 的子图通过一系列的 边收缩而得到, 则称 $H$ 是 $G$ 的图子式. 若 $H$ 不是 $G$ 的图子式, 则称 $G$ 没有 $H$-图子式. 设 $V(G)$ 和 $E(G)$ 分别表示图 $G$ 的顶点集合和边集合. 定义 $V E(G)=V(G) \cup E(G)$. G 的一个 正常 $k$-全染色是一个映射 $\phi: V E(G) \rightarrow\{1,2, \ldots, k\}$, 使得 $V E(G)$ 中任意一对相邻或相关联 的元素均得到不同色. $G$ 的全色数 $\chi^{\prime \prime}(G)$ 是使得 $G$ 有一个 $k$-全染色的最小整数 $k$. 设

$$
C_{\phi}(v)=\{\phi(x v) \mid x v \in E(G)\} \cup\{\phi(v)\}
$$

表示顶点 $v$ 和它的关联边所指定的颜色集合. 若对任一条边 $u v \in E(G)$ 有 $C_{\phi}(u) \neq C_{\phi}(v)$, 则称 $G$ 的一个正常 $k$-全染色 $\phi$ 是邻点可区别的, 或称 $\phi$ 是 $G$ 的一个 $k$-邻点可区别全染色. $G$ 的邻点可区别全色数 $\chi_{a}^{\prime \prime}(G)$ 是使得 $G$ 有一个 $k$-邻点可区别全染色的最小的整数 $k$. 同理 可以给出图 $G$ 的邻点可区别边色数 $\chi_{a}^{\prime}(G)$ 的定义.

图的邻点可区别边染色是点可区别边染色的特殊情形. 若图 $G$ 的任意一对顶点的颜色 集合均不相同, 则称 $G$ 有一个点可区别边染色. 这一概念早在 20 年前就已给出, 至今多篇研 究论文已经发表. 张忠辅等人 ${ }^{[1]}$ 提出下面猜想: 
猜想 $\mathbf{1}^{[1]}$ 如果 $G$ 是一个连通的简单图且 $G \neq C_{5}$ 和 $G \neq K_{2}$, 则 $\chi_{a}^{\prime}(G) \leqslant \Delta(G)+2$. 以下两个定理是关于图的邻点可区别边染色的最新结果：

定理 $\mathbf{1}^{[2]}$ 如果 $G$ 是一个没有孤立边的 $k$-色图, 则 $\chi_{a}^{\prime}(G) \leqslant \Delta(G)+O(\log k)$.

定理 $2^{[3]}$ 如果 $G$ 是一个没有孤立边且 $\Delta(G) \geqslant 10^{20}$ 的图, 则 $\chi_{a}^{\prime}(G) \leqslant \Delta(G)+300$.

图的点可区别全染色是点可区别边染色的另外一种推广. 根据定义, $\chi_{a}^{\prime \prime}(G) \geqslant \chi^{\prime \prime}(G)$ $\geqslant \Delta(G)+1$ 是平凡的. 下面著名的全染色猜想是由 Behzad ${ }^{[4]}$ 和 Vizing ${ }^{[5]}$ 独立提出的.

猜想 2 对任意的简单图 $G$, 有 $\chi^{\prime \prime}(G) \leqslant \Delta(G)+2$.

对应地, 张忠辅等人 ${ }^{[6]}$ 提出了下面猜想.

猜想 3 对每一个 $|V(G)| \geqslant 2$ 的图 $G$, 有 $\chi_{a}^{\prime \prime}(G) \leqslant \Delta(G)+3$.

注意到 $\chi_{a}^{\prime \prime}\left(K_{2 n+1}\right)=\Delta\left(K_{2 n+1}\right)+3=2 n+3$, 其中 $n \geqslant 1$. 这个例子说明如果猜想 3 成 立, 则 $\chi_{a}^{\prime \prime}(G)$ 的上界 $\Delta(G)+3$ 是紧的. 在文献 [6] 中, 作者确定了路、圈、扇、轮、树、完全 图、完全二部图的邻点可区别全色数. Chen ${ }^{[7]}$ 和 $\mathrm{Wang}^{[8]}$ 分别独立地证明了猜想 3 对最大 度至多为 3 的图成立. 文献 [9] 完整刻画了外可平面图的邻点可区别全色数. 本文的主要结 果如下:

定理 3 (主要定理) 设 $G$ 是一个没有 $K_{4}$-图子式的图且 $\Delta(G) \geqslant 3$. 则 $\Delta(G)+1 \leqslant$ $\chi_{a}^{\prime \prime}(G) \leqslant \Delta(G)+2$, 且 $\chi_{a}^{\prime \prime}(G)=\Delta(G)+2$ 当且仅当 $G$ 含有两个相邻最大度点.

注意到, 一个图是外可平面图当且仅当它不含 $K_{2,3}$-图子式和 $K_{4}$-图子式. 故外可平面 图是没有 $K_{4}$-图子式的图的子类. 因此, 本文的主要定理推广了文献 [9] 中的结论.

\section{2 没有 $K_{4}$-图子式的图的结构性质}

假设 $G$ 是一个没有 $K_{4}$-图子式的图. 设 $u \in V(G)$. 若 $u$ 的度数是 $k$ (至少 $k$, 至多 $k$ ), 则称 $u$ 是一个 $k$-点 ( $k^{+}$-点, $k^{-}$-点). 对 $i \geqslant 1$, 用 $n_{i}(u)$ 表示与 $u$ 相邻的 $i$-点的个数. $u$ 被称 作一个中心点, 如果对任意的 $v \in N_{G}(u)$, 或者 $d(v) \geqslant 3$, 或者 $d(v)=2$ 且 $v$ 相邻于 $N_{G}(u)$ 中的另一个 $3^{+}$-点. 对一个中心点 $u$, 定义 $S(u)=\left\{x \in N_{G}(u) \mid d(x) \geqslant 3\right\}$ 和 $D_{G}(u)=|S(u)|$.

引理 $\mathbf{1}^{[10]}$ 每一个 $\Delta(G) \geqslant 3, \delta(G)=2$, 没有 $K_{4}$-图子式的图 $G$ 含有两个相邻的 2-点, 或者含有子图形 $\left(\mathrm{C}^{*}\right)$, 其中 $\left(\mathrm{C}^{*}\right)$ 是一个中心点 $u$, 满足 $d(u) \geqslant 3$ 和 $D_{G}(u) \leqslant 2$.

引理 2 每一个连通的 $\Delta(G) \geqslant 3$ 的没有 $K_{4}$-图子式的图 $G$ 含有下面子图形 $(\mathrm{C} 1)-(\mathrm{C} 6)$ 之一:

(C1) 一个顶点相邻于至少一个叶子和至多两个 $3^{+}$-点.

$(\mathrm{C} 2)$ 一条路 $x_{1} x_{2} \cdots x_{m}, m \geqslant 4$, 满足 $d\left(x_{1}\right) \geqslant 3, d\left(x_{m}\right) \geqslant 2, d\left(x_{i}\right)=2, i=2,3, \ldots, m-1$.

(C3) 一个 3 -圈 $x y z$ 满足 $d(z)=2$ 和 $d(x)=3$.

(C4) 两个 3 -圈 $x x_{1} z$ 和 $y y_{1} z$ 满足 $d(z)=4$ 和 $d\left(x_{1}\right)=d\left(y_{1}\right)=2$.

(C5) 一个 4-圈 $y_{1} y_{2} y_{3} y_{4}$ 使得 $d\left(y_{2}\right)=d\left(y_{4}\right)=2, d\left(y_{1}\right), d\left(y_{3}\right) \geqslant 3$, 且 $y_{1}$ 至多与两个 $3^{+}$-点相邻.

(C6) 一个 3 -圈 $x y z$ 使得 $d(x) \geqslant 3$ 和 $d(y)=d(z)=2$.

证明 假设 $G$ 不含 $(\mathrm{C} 1)$, 于是不存在与叶子相邻的 $3^{-}$点. 令 $H$ 是去掉 $G$ 中所有叶子 后所得到的图. 显然, $H$ 仍旧是一个没有 $K_{4}$-图子式的图. 如果 $\delta(H)=1$, 则 $G$ 中必存在一 点 $u$, 使得它的邻点至多一个除外其余都是叶子, 即 $G$ 包含 $(\mathrm{C} 1)$, 矛盾. 于是 $\delta(H) \geqslant 2$. 因 
为 $G$ 不含 $(\mathrm{C} 1)$, 对任意满足 $2 \leqslant d_{G}(v) \leqslant 3$ 的顶点 $v$ 必有 $d_{H}(v)=d_{G}(v)$. 推出 $\Delta(H) \geqslant 3$. 根据引理 $1, H$ 或者含有一对相邻 2 -点, 或者含有 $\left(\mathrm{C}^{*}\right)$. 如果 $H$ 含有一对相邻 2-点, 则考虑 这对相邻 2-点的邻点. 如果它们至少之一是 $3^{+}$-点且二者互异, 则 $(\mathrm{C} 2)$ 在 $H$ 中出现, 因此 也在 $G$ 中出现. 如果二者是重合的 $3^{+}$-点, 则 (C6) 在 $G$ 中出现. 如果它们都是 2-点, 由于 $\Delta(H) \geqslant 3$ 且 $H$ 是连通图, 重复这一过程直到 $3^{+}$点出现, 归结到前面情形.

如果 $H$ 含有 $\left(\mathrm{C}^{*}\right)$, 即一个满足 $d_{H}(u) \geqslant 3$ 和 $\left|S_{H}(u)\right| \leqslant 2$ 的点 $u \in V(H)$. 注意到, $u$ 在 $H$ 中至多相邻两个度至少 3 的顶点. 因为 (C1) 不在 $G$ 中出现, $G$ 中没有叶子与 $u$ 相邻. 因此, $d_{G}(u)=d_{H}(u)$. 另一方面, 容易看到, $\left|S_{G}(u)\right|=\left|S_{H}(u)\right| \leqslant 2$. 因此, $\left(\mathrm{C}^{*}\right)$ 也在 $G$ 中出 现. 如果 $\left|S_{H}(u)\right|=1$, 则 $S_{G}(u)=\{x\}$. 因为 $d_{G}(u) \geqslant 3$, 所以必存在两个 2 -点 $x_{1}, x_{2}$ 使得 它们同时与 $u$ 和 $x$ 相邻. 因此, $u x_{1} x x_{2}$ 形成了一个 4 -圈, 即 (C5) 出现. 假设 $\left|S_{G}(u)\right|=2$ 和 $S_{G}(u)=\{x, y\}$. 如果存在两个 2 -点使其或者同时与 $u$ 和 $x$ 相邻或者同时与 $u$ 和 $y$ 相邻, $(\mathrm{C} 5)$ 再次在 $G$ 中出现. 否则, 容易看出 $d_{G}(u) \leqslant 4$. 如果 $d_{G}(u)=3,(\mathrm{C} 3)$ 在 $G$ 中出现. 如果 $d_{G}(u)=4,(\mathrm{C} 4)$ 在 $G$ 中出现. 引理证毕.

如果一个 3 -点恰与一个叶子相邻, 则称之为可控制点.

引理 3 每一个连通的 $\Delta(G)=3$ 的没有 $K_{4}$-图子式的图 $G$ 含有下列结构之一:

(B1) 一个顶点 $v$ 相邻于至少 $d(v)-1$ 个叶子.

(B2) 一条迹 $P=x_{1} x_{2} x_{3} x_{4}$ 满足 $d\left(x_{1}\right)=3, d\left(x_{4}\right) \geqslant 2, x_{2}$ 和 $x_{3}$ 或者是 2 -点或者是可控 制 3-点.

(B3) 一个 3 -圈 $x y z$ 使得 $d(x)=3, z$ 或是 2 -点或是可控制 3 -点.

(B4) 一个 4-圈 $y_{1} y_{2} y_{3} y_{4}$ 使得 $y_{2}$ 和 $y_{4}$ 或是 2-点或是可控制 3 -点.

证明 假设 $G$ 不含 (B1)-(B4). 令 $H$ 是由 $G$ 去掉所有叶子后所得到的图. 如果存在 $v \in V(H)$ 使得 $d_{H}(v)=1$, 则 $G$ 必含有 (B1), 矛盾. 如果 $\Delta(H)=2$, 则 $H$ 是一个圈. 因为 $\Delta(G)=3, G$ 必含有 (B2), 矛盾. 因此, 假设 $\delta(H) \geqslant 2$ 和 $\Delta(H)=3$. 根据引理 $2, H$ 必含有 $(\mathrm{C} 2) 、(\mathrm{C} 3) 、(\mathrm{C} 5) 、(\mathrm{C} 6)$ 结构之一. 注意到在 $(\mathrm{C} 6)$ 中 $d_{H}(x)$ 不能大于 3 , 所以只需考虑 $(\mathrm{C} 3)$. 如果 $H$ 含有 $(\mathrm{C} 2),(\mathrm{C} 3)$ 或 (C5), 则 $G$ 分别含有 (B2)、(B3) 或 (B4), 矛盾. 引理证毕.

注意到在 (B2) 中 $x_{1}$ 可能与 $x_{4}$ 重合. 如果 $x_{1}=x_{4}$, 只有在 $\Delta(G) \geqslant 4$ 时 (B3) 与 (B2) 的结构不同.

推论 1 设 $G$ 是一个连通的 $\Delta(G)=3$ 的没有 $K_{4}$-图子式的图. 如果 $G$ 不含相邻 3-点, 则 $G$ 含有下列子图形之一:

(A1) 一个叶子.

(A2) 两个相邻 2-点.

(A3) 一个 4 -圈 $y_{1} y_{2} y_{3} y_{4}$ 满足 $d\left(y_{1}\right)=d\left(y_{3}\right)=3$ 和 $d\left(y_{2}\right)=d\left(y_{4}\right)=2$.

引理 4 设 $G$ 是一个连通的 $\Delta(G)=4$ 的没有 $K_{4}$-图子式的图. 如果 $G$ 不含相邻 4-点, 则 $G$ 含有 (B1)、(B2)、(B3)、(B4) 结构之一, 其中 (B1) 是一个顶点 $u$, 满足或者 $d(u) \neq 3$ 且 $u$ 相邻于一个叶子, 或者 $d(u)=3$ 且 $n_{3}(u) \leqslant 1$.

证明 假设 $G$ 不含 (B1)-(B4). 令 $H$ 是由 $G$ 去掉所有叶子后所得到的图. 如果 $H$ 中存 在一个叶子 $v$, 则在 $G$ 中 $n_{3}(v) \leqslant 1$ 并且 $v$ 至少与 $d_{G}(v)-1$ 个叶子相邻, 推出 $G$ 含有 (B1), 矛盾. 根据引理 $2, H$ 含有 $(\mathrm{C} 2)-(\mathrm{C} 6)$ 结构之一. 如果 $H$ 含有 $(\mathrm{C} 2)$ 或 $(\mathrm{C} 3)$, 则 $G$ 分别含有 
(B2) 或 (B3), 矛盾. 如果 $H$ 含有 (C4), 则 $x$ 和 $y$ 的度数都不为 4 , 因为 $d_{H}(z)=d_{G}(z)=4$ 且 $G$ 不含相邻 4-点. 推出 $G$ 含有 (B3), 矛盾. 如果 $H$ 含有 (C5) 或 (C6), 则 $G$ 分别含有 (B4) 或 (B2), 矛盾. 证毕.

\section{3 预备引理}

我们用归纳法来证明主要定理. 假设 $H \subset G, \phi$ 是 $H$ 的一个 $k$-邻点可区别全染色, $\Phi$ 是 $G$ 的一个 $k$-邻点可区别全染色, $\phi$ 和 $\Phi$ 应用相同的颜色集合 $C=\{1,2, \ldots, k\}, k \geqslant 5$. 设 $v$ 是一个 $2^{-}$-点并且不与自己度数相同的顶点相邻. 因为 $v$ 至多有 2 个邻点和 2 条关联边且 $|C| \geqslant 5$, 我们可以把 $v$ 放到最后去染, 即当 $v$ 的所有邻点和关联边都染好之后, 再去染 $v$. 不 过, 我们经常省略这一步骤. 当把一个邻点可区别全染色 $\phi$ 从 $H$ 扩展到 $G$ 时, 我们经常说 “染一个顶点” 或 “染一条边”, 即使那个点或边已经被染色了. 换言之, 我们先去掉这个顶点 或边的颜色, 然后再补染它们. “在一对相邻顶点之间有着潜在的矛盾” 意味着对 $u v \in E(G)$ 存在着 $C_{\phi}(u)=C_{\phi}(v)$ 的可能性.

引理 $5^{[6]}$ 设 $G$ 是一个 $\Delta(G) \leqslant 2$ 的图, 则 $\chi_{a}^{\prime \prime}(G) \leqslant 5$, 且 $\chi_{a}^{\prime \prime}(G)=5$ 当且仅当 $G$ 是一 个 3 -圈.

引理 $\mathbf{6}^{[6]}$ 如果 $G$ 是一个含有相邻最大度点的图, 则 $\chi_{a}^{\prime \prime}(G) \geqslant \Delta(G)+2$.

引理 7 设一个图 $G$ 含有一个 3 -点 $u$ 相邻于一个叶子 $v$. 假设 $G-v$ 有一个 $k$-邻点可 区别全染色 $\phi$ 应用色集 $C=\{1,2, \ldots, k\}$. 如果下面条件之一成立, 则 $\phi$ 能被延拓到 $G$ 上:

(1) $k \geqslant 6$; 或

(2) $k=5$ 且 $n_{3}(u) \leqslant 1$.

证明 令 $u_{1}, u_{2}$ 是 $u$ 的不同于 $v$ 的邻点. 不妨设 $\phi(u)=1, \phi\left(u u_{1}\right)=2, \phi\left(u u_{2}\right)=3$.

首先假设 (1) 成立, 即 $|C|=k \geqslant 6$. 设 $c \in\{4,5,6\}$. 如果 $c \in C_{\phi}\left(u_{1}\right) \cap C_{\phi}\left(u_{2}\right)$, 我们 可以给 $u v$ 染 $\{4,5,6\} \backslash\{c\}$ 中的某种颜色. 如果 $c \notin C_{\phi}\left(u_{1}\right) \cup C_{\phi}\left(u_{2}\right)$, 令 $\Phi(u v)=c$. 如果 $\{4,5,6\} \subseteq C_{\phi}\left(u_{1}\right) \backslash C_{\phi}\left(u_{2}\right)$ 或 $\{4,5,6\} \subseteq C_{\phi}\left(u_{2}\right) \backslash C_{\phi}\left(u_{1}\right)$, 令 $\Phi(u v)=4$. 余下要考虑的是 $\{4,5,6\}$ 中的有两种颜色或者在 $C_{\phi}\left(u_{1}\right) \backslash C_{\phi}\left(u_{2}\right)$ 中, 或者在 $C_{\phi}\left(u_{2}\right) \backslash C_{\phi}\left(u_{1}\right)$ 中. 不失一般性, 假设 $4,5 \in C_{\phi}\left(u_{1}\right) \backslash C_{\phi}\left(u_{2}\right)$ 且 $6 \in C_{\phi}\left(u_{2}\right) \backslash C_{\phi}\left(u_{1}\right)$. 令 $\Phi(u v)=4$. 最后, 我们给 $v$ 正常染色. 容易检验 $C_{\Phi}(u) \neq C_{\Phi}\left(u_{i}\right), i=1,2$.

其次假设 $(2)$ 成立. 因为 $n_{3}(u) \leqslant 1$, 可以假设 $d\left(u_{2}\right) \neq 3$. 如果 4,5 之一不属于 $C_{\phi}\left(u_{1}\right)$, 比如 $5 \notin C_{\phi}\left(u_{1}\right)$, 我们给 $u v$ 染 5 . 否则, 给 $u v$ 染 4 . 显然, $C_{\Phi}(u) \neq C_{\Phi}\left(u_{1}\right)$. 引理证毕.

引理 8 令 $v$ 是图 $G$ 的一个 $m$-点, $m \geqslant 4, v_{1}, v_{2}, \ldots, v_{m}$ 是它的邻点使得 $d\left(v_{1}\right)=1$, $d\left(v_{i}\right) \leqslant 2, i=2,3, \ldots, m-2$. 假设 $G-v_{1}$ 有一个 $k$-邻点可区别全染色 $\phi$ 应用色集 $C=$ $\{1,2, \ldots, k\}$. 如果下面条件之一成立, 则 $\phi$ 能被延拓到 $G$ 上:

(1) $k \geqslant m+2 ;$ 或

(2) $k=m+1$ 且 $n_{m}(v)=0$.

证明 如果 $d\left(v_{i}\right)=2$, 设 $v_{i}^{\prime}$ 是 $v_{i}$ 的不同于 $v$ 的邻点, $i=2, \ldots, m-2$. 不失一般性, 假 设 $\phi(v)=1, \phi\left(v v_{i}\right)=i, i=2,3, \ldots, m$.

(1) 若 $m+1 \in C_{\phi}\left(v_{m-1}\right) \cap C_{\phi}\left(v_{m}\right)$, 我们给 $v v_{1}$ 染 $m+2$, 然后给 $v_{1}$ 正常染色. 若 $m+2 \in C_{\phi}\left(v_{m-1}\right) \cap C_{\phi}\left(v_{m}\right)$, 有类似的讨论. 如果 $m+1 \notin C_{\phi}\left(v_{m-1}\right) \cup C_{\phi}\left(v_{m}\right)$, 则给 $v v_{1}$ 染 $m+1$. 可能的冲突会发生在 $v$ 和 $v_{m-1}$ 之间或 $v$ 和 $v_{m}$ 之间. 然而, $m+1$ 属于 $C_{\Phi}\left(v_{m-1}\right)$ 
和 $C_{\Phi}\left(v_{m}\right)$, 不属于 $C_{\Phi}(v)$, 故 $\Phi$ 是 $G$ 的一个正常邻点可区别全染色. 同样可以处理 $m+2 \notin$ $C_{\phi}\left(v_{m-1}\right) \cup C_{\phi}\left(v_{m}\right)$ 的情形.

如果 $\{m+1, m+2\} \subseteq C_{\phi}\left(v_{m-1}\right) \backslash C_{\phi}\left(v_{m}\right)$ 或 $\{m+1, m+2\} \subseteq C_{\phi}\left(v_{m}\right) \backslash C_{\phi}\left(v_{m-1}\right)$, 则 给 $v v_{1}$ 染 $m+1$. 接下来, 假设 $m+1 \in C_{\phi}\left(v_{m-1}\right) \backslash C_{\phi}\left(v_{m}\right)$ 和 $m+2 \in C_{\phi}\left(v_{m}\right) \backslash C_{\phi}\left(v_{m-1}\right)$. 如果 $d\left(v_{2}\right)=1$, 则给 $v v_{2}$ 改染 $m+1, v v_{1}$ 染 $m+2$. 如果 $d\left(v_{2}\right)=2$, 给 $v v_{2}$ 改染 $a \in$ $\{m+1, m+2\} \backslash\left\{\phi\left(v_{2} v_{2}^{\prime}\right)\right\}, v v_{1}$ 染 $\{m+1, m+2\} \backslash\{a\}$ 中的颜色, $v_{2}$ 染 $C \backslash\left\{1, a, \phi\left(v_{2}^{\prime}\right), \phi\left(v_{2}^{\prime} v_{2}\right)\right\}$ 中的颜色.

(2) 因为 $n_{m}(v)=0, v$ 不相邻于任何 $m$-点. 只需给 $v v_{1}$ 染 $m+1$. 证毕.

引理 9 令 $v$ 是图 $G$ 的一个 $m$-点, $m \geqslant 3, v_{1}, v_{2}, \ldots, v_{m}$ 是它的邻点使得 $d\left(v_{1}\right)=$ $d\left(v_{2}\right)=2, d\left(v_{i}\right) \leqslant 2, i=3, \ldots, m-2$, 且 $v_{1}$ 和 $v_{2}$ 同时与一个 $3^{+}$-点 $u$ 相邻. 假设 $G-v v_{1}$ 有 一个 $k$-邻点可区别全染色 $\phi$ 应用色集 $C=\{1,2, \ldots, k\}$. 如果下面条件之一成立, 则 $\phi$ 能被 延拓到 $G$ 上:

(1) $k \geqslant m+2$; 或

(2) $k=m+1$ 且 $n_{m}(v)=0$.

证明 假设 $\phi(v)=1, \phi\left(v v_{i}\right)=i, i=2,3, \ldots, m$. 因为 $v_{1}, v_{2}$ 与 $u$ 相邻, 则有 $\phi\left(u v_{1}\right)$ $\neq \phi\left(u v_{2}\right)$.

(1) 抹去 $u v_{1}$ 和 $u v_{2}$ 的颜色. 类似于引理 8 的讨论, 或者给 $v v_{1}$ 染 $\{m+1, m+2\}$ 中的 某色, 或者给 $v v_{1}$ 和 $v v_{2}$ 染 $\{m+1, m+2\}$ 中的不同颜色. 如果 $u v_{1}$ 和 $v v_{1}$ 得到相同的颜 色, 或者 $u v_{2}$ 和 $v v_{2}$ 得到相同的颜色, 我们需要交换 $v v_{1}$ 和 $v v_{2}$ 的颜色. 因为颜色 $2 、 m+1$ (或 $m+2) 、 \phi\left(u v_{2}\right)$ 互不相同, 所以这种交换是可行的. 容易验证 $C_{\Phi}(v) \neq C_{\Phi}\left(v_{m-1}\right)$ 和 $C_{\Phi}(v) \neq C_{\Phi}\left(v_{m}\right)$.

(2) 因为 $n_{m}(v)=0, v$ 不与任何 $m$-点相邻. 没有潜在的冲突存在. 只需给 $v v_{1}$ 染 $m+1$. 当 $\phi\left(u v_{1}\right)=m+1$ 时, 需要进一步交换 $v v_{1}$ 和 $v v_{2}$ 的颜色. 证毕.

\section{4 上界 $\Delta+2$}

本节建立了 $\chi_{a}^{\prime \prime}(G)$ 的上界.

定理 4 如果 $G$ 是一个 $\Delta(G) \geqslant 3$ 且没有 $K_{4}$-图子式的图, 则 $\chi_{a}^{\prime \prime}(G) \leqslant \Delta(G)+2$.

证明 对 $|V E(G)|$ 进行归纳法证明. 如果 $|V E(G)| \leqslant 7$, 定理显然成立. 假设 $G$ 是一个 连通的没有 $K_{4}$-图子式的图且 $\Delta(G) \geqslant 3$ 和 $|V E(G)| \geqslant 8$. 对于一个 $\Delta(H) \leqslant 2$ 且不同于 3 -圈 的图 $H$, 由引理 5 , 定理成立. 因此, 我们总假设 $\Delta(H) \geqslant 3$. 由归纳假设, 每一个没有 $K_{4}$-图 子式的图 $H$ 且满足 $\Delta(H) \leqslant \Delta(G)$ 和 $|V E(H)|<|V E(G)|$ 有 $\chi_{a}^{\prime \prime}(H) \leqslant \Delta(H)+2 \leqslant \Delta(G)+2$. 证明分两个部分.

\section{第 1 部分 $\Delta(G)=3$.}

根据引理 $3, G$ 含有 (B1)-(B4) 结构之一, 我们分别加以处理.

(B1) 存在一个顶点 $v$ 相邻于至少 $d(v)-1$ 个叶子.

假设 $v_{1}, \ldots, v_{m}$ 是 $v$ 的邻点使得 $d\left(v_{1}\right)=\cdots=d\left(v_{m-1}\right)=1, d\left(v_{m}\right) \geqslant 1$. 显然, $2 \leqslant$ $m \leqslant 3$. 令 $H=G-\left\{v_{1}, \ldots, v_{m-1}\right\}$, 则 $H$ 是一个没有 $K_{4}$-图子式的图, 满足 $|V E(H)|<$ $|V E(G)|$. 由归纳假设, $H$ 有一个 5 -邻点可区别全染色 $\phi$ 应用色集 $C=\{1,2, \ldots, 5\}$. 我 
们能给 $v v_{1}, \ldots, v v_{m-1}$ 染集合 $C \backslash\left\{\phi(v), \phi\left(v_{m}\right), \phi\left(v v_{m}\right)\right\}$ 中的不同颜色. 因为 $m-1 \leqslant 2$, $\left|C \backslash\left\{\phi(v), \phi\left(v_{m}\right), \phi\left(v v_{m}\right)\right\}\right| \geqslant 2$, 所以 $\Phi$ 是 $G$ 的一个 5 -邻点可区别全染色.

(B2) 存在一条迹 $x_{1} x_{2} x_{3} x_{4}$ 使得 $d\left(x_{1}\right)=3, x_{2}$ 和 $x_{3}$ 或是 2-点或是可控制 3 -点.

对 $i \in\{2,3\}$, 若 $d\left(x_{i}\right)=3$, 令 $x_{i}^{\prime}$ 是一个与 $x_{i}$ 相邻的叶子. 需要考虑以下 4 种情形.

(B2.1) $d\left(x_{2}\right)=d\left(x_{3}\right)=2$.

令 $H=G-x_{2} x_{3}$. 由归纳假设, $H$ 有一个 5 -邻点可区别全染色 $\phi$ 应用色集 $C=$ $\{1,2, \ldots, 5\}$. 如果 $d\left(x_{4}\right)=3$, 我们给 $x_{2}$ 染 $a \in C \backslash\left\{\phi\left(x_{1}\right), \phi\left(x_{3}\right), \phi\left(x_{1} x_{2}\right), \phi\left(x_{3} x_{4}\right)\right\}$, 给 $x_{2} x_{3}$ 染 $C \backslash\left\{a, \phi\left(x_{3}\right), \phi\left(x_{1} x_{2}\right), \phi\left(x_{3} x_{4}\right)\right\}$ 中的颜色. 很容易验证 $C_{\Phi}\left(x_{2}\right) \neq C_{\Phi}\left(x_{3}\right)$.

若 $d\left(x_{4}\right)=2$, 令 $x_{5} \neq x_{3}$ 是 $x_{4}$ 的邻点. 给 $x_{3} x_{4}$ 改染 $a \in C \backslash\left\{\phi\left(x_{2}\right), \phi\left(x_{4}\right), \phi\left(x_{5}\right), \phi\left(x_{4} x_{5}\right)\right\}$, $x_{3}$ 染 $b \in C \backslash\left\{a, \phi\left(x_{2}\right), \phi\left(x_{4}\right), \phi\left(x_{4} x_{5}\right)\right\}, x_{2} x_{3}$ 染 $C \backslash\left\{a, b, \phi\left(x_{2}\right), \phi\left(x_{1} x_{2}\right)\right\}$ 中的颜色. 则 $C_{\Phi}\left(x_{2}\right)=\left\{\phi\left(x_{2}\right), \Phi\left(x_{2} x_{3}\right), \phi\left(x_{1} x_{2}\right)\right\}, C_{\Phi}\left(x_{3}\right)=\left\{a, b, \Phi\left(x_{2} x_{3}\right)\right\}, C_{\Phi}\left(x_{4}\right)=\left\{a, \phi\left(x_{4} x_{5}\right), \phi\left(x_{4}\right)\right\}$. 因为 $\phi\left(x_{2}\right) \notin\left\{a, b, \Phi\left(x_{2} x_{3}\right)\right\}$, 推出 $C_{\Phi}\left(x_{2}\right) \neq C_{\Phi}\left(x_{3}\right)$; 因为 $b \notin\left\{a, \phi\left(x_{4} x_{5}\right), \phi\left(x_{4}\right)\right\}$, 推出 $C_{\Phi}\left(x_{3}\right) \neq C_{\Phi}\left(x_{4}\right)$.

(B2.2) $d\left(x_{2}\right)=d\left(x_{3}\right)=3$.

令 $H$ 是重合 $G$ 中顶点 $x_{2}^{\prime}$ 和 $x_{3}^{\prime}$ 后得到图. 则 $H$ 是一个没有 $K_{4}$-图子式的图, $\Delta(H) \leqslant 3$, $|V E(H)|=|V E(G)|-1$. 显然, $H$ 的任意 5-邻点可区别全染色可以扩展到 $G$ 上.

(B2.3) $d\left(x_{2}\right)=3$ 且 $d\left(x_{3}\right)=2$.

图 $G-x_{2}^{\prime}$ 有一个 5 -邻点可区别全染色 $\phi$ 应用 $C=\{1,2, \ldots, 5\}$. 假设 $C_{\phi}\left(x_{1}\right)=\{1,2,3,4\}$. 如果 $5 \in\left\{\phi\left(x_{2}\right), \phi\left(x_{2} x_{3}\right)\right\}$, 只需给 $x_{2} x_{2}^{\prime}$ 正常染色. 否则, 给 $x_{2} x_{2}^{\prime}$ 染 5 .

(B2.4) $d\left(x_{2}\right)=2$ 且 $d\left(x_{3}\right)=3$.

图 $G-x_{3}^{\prime}$ 有一个 5 邻点可区别全染色 $\phi$ 应用 $C=\{1,2, \ldots, 5\}$. 如果 $d\left(x_{4}\right)=2$, 给 $x_{3} x_{3}^{\prime}$ 正常染色. 否则, 假设 $C_{\phi}\left(x_{4}\right)=\{1,2,3,4\}$. 如果 $5 \in\left\{\phi\left(x_{3}\right), \phi\left(x_{2} x_{3}\right)\right\}$, 给 $x_{3} x_{3}^{\prime}$ 正常染色. 如 果 $5 \notin\left\{\phi\left(x_{3}\right), \phi\left(x_{2} x_{3}\right)\right\}$, 给 $x_{3} x_{3}^{\prime}$ 染 5 .

(B3) 存在一个 3 -圈 $x y z$ 使得或 $d(z)=2$, 或 $d(z)=3$ 且 $z$ 与一个叶子 $z^{\prime}$ 相邻.

基于 (B2.2) 的证明, 我们假设 $d(x)=d(y)=3$. 令 $x^{\prime} \neq y, z$ 是 $x$ 的第 3 个邻点, $y^{\prime} \neq x, z$ 是 $y$ 的第 3 个邻点.

(B3.1) $x^{\prime}$ 与 $y^{\prime}$ 重合. 设 $\phi$ 是 $G-\{z, x y\}$ 的 5-邻点可区别全染色应用 $C=\{1,2, \ldots, 5\}$. 若 $d\left(x^{\prime}\right)=2$, 即 $G$ 的阶数至多为 5 , 定理显然成立. 假设 $d\left(x^{\prime}\right)=3$, 且 $x^{\prime \prime} \neq x, y$ 是 $x^{\prime}$ 的第 3 个邻点. 不失一般性, 假设 $\phi\left(x^{\prime} x^{\prime \prime}\right)=1, \phi\left(x^{\prime}\right)=2, \phi\left(x^{\prime} x\right)=3, \phi\left(x^{\prime} y\right)=4$. 给 $\left\{z^{\prime}, y z\right\}$ 染 1 (若 $z^{\prime}$ 存在), $x z$ 染 $2,\left\{y, z z^{\prime}\right\}$ 染 3 (若 $z^{\prime}$ 存在), $x$ 染 $4,\{z, x y\}$ 染 5 .

(B3.2) $x^{\prime} \neq y^{\prime}$ 且 $x^{\prime} y^{\prime} \in E(G)$. 只需考虑 $x^{\prime}$ 和 $y^{\prime}$ 都为 3 -点时的困难情形. 令 $x^{\prime \prime} \neq x, y^{\prime}$ 是 $x^{\prime}$ 的第 3 个邻点, $y^{\prime \prime} \neq y, x^{\prime}$ 是 $y^{\prime}$ 的第 3 个邻点. 图 $G-\{z, x y\}$ 有一个 5 -邻点可区 别全染色 $\phi$ 应用 $C=\{1,2, \ldots, 5\}$. 不失一般性, 假设 $\phi\left(x^{\prime}\right)=1, \phi\left(x^{\prime} y^{\prime}\right)=2, \phi\left(x^{\prime} x^{\prime \prime}\right)=3$, $\phi\left(x^{\prime} x\right)=4$. 因为 $C_{\phi}\left(x^{\prime}\right) \neq C_{\phi}\left(y^{\prime}\right)$, 颜色 5 必在 $\left\{\phi\left(y^{\prime}\right), \phi\left(y y^{\prime}\right), \phi\left(y^{\prime} y^{\prime \prime}\right)\right\}$ 中.

(B3.2.1) $\phi\left(y^{\prime}\right)=5$. 则 $\phi\left(y y^{\prime}\right) \in\{1,3,4\}$. 首先, 给 $\{y, x z\}$ 染 $2,\left\{x, z z^{\prime}\right\}$ 染 5 (若 $z^{\prime}$ 存 在). 其次, 根据 $\phi\left(y y^{\prime}\right)$ 的值来染剩下的元素. 若 $\phi\left(y y^{\prime}\right)=1$, 给 $z$ 染 $1, x y$ 染 $3, y z$ 染 4 . 若 $\phi\left(y y^{\prime}\right)=3$, 给 $x y$ 染 $1, z$ 染 $3, y z$ 染 4 . 若 $\phi\left(y y^{\prime}\right)=4$, 给 $y z$ 染 $1,\{z, x y\}$ 染 3 .

(B3.2.2) $\phi\left(y y^{\prime}\right)=5$. 给 $\left\{y, z z^{\prime}\right\}$ 染 1 (若 $z^{\prime}$ 存在), $x z$ 染 $2,\{z, x y\}$ 染 $3, y z$ 染 4 , 给 $x$ 染 5 (若 $z^{\prime}$ 存在). 
(B3.2.3) $\phi\left(y^{\prime} y^{\prime \prime}\right)=5$. 则 $\phi\left(y y^{\prime}\right) \in\{1,3,4\}$. 首先给 $\{y, x z\}$ 染 $2,\left\{x, z z^{\prime}\right\}$ 染 5 (若 $z^{\prime}$ 存 在). 若 $\phi\left(y y^{\prime}\right)=1$, 给 $z$ 染 $1, x y$ 染 $3, y z$ 染 4 . 若 $\phi\left(y y^{\prime}\right)=3$, 给 $x y$ 染 $1, z$ 染 $3, y z$ 染 4 . 若 $\phi\left(y y^{\prime}\right)=4$, 给 $x y$ 染 $1, y z$ 染 $3, z$ 染 4 .

(B3.3) $x^{\prime} \neq y^{\prime}$ 且 $x^{\prime} y^{\prime} \notin E(G)$. 令 $H=G-\{x, y, z\}+x^{\prime} y^{\prime}$. 显然, $H$ 是一个没有 $K_{4}$-图子 式的图且 $|V E(H)|<|V E(G)|$, 因此, 它有一个 5-邻点可区别全染色 $\phi$ 应用 $C=\{1,2, \ldots, 5\}$. 不失一般性, 假设 $\phi\left(x^{\prime}\right)=1, \phi\left(x^{\prime} y^{\prime}\right)=2, \phi\left(y^{\prime}\right)=3$. 在 $G$ 中, 给 $\left\{y, z z^{\prime}\right\}$ 染 1 (若 $z^{\prime}$ 存在), $\left\{z, x x^{\prime}, y y^{\prime}\right\}$ 染 $2, x z$ 染 $3,\{x, y z\}$ 染 $4, x y$ 染 5 .

(B4) 存在一个 4 -圈 $y_{1} y_{2} y_{3} y_{4}$ 使得 $y_{2}$ 和 $y_{4}$ 或者是 2-点或者是可控制 3-点.

对 $i=2,4$, 若 $d\left(y_{i}\right)=3$, 令 $y_{i}^{\prime}$ 是与 $y_{i}$ 相邻的叶子. 由 (B2) 的证明, 可以假设 $y_{1}$ 和 $y_{3}$ 都是 3 -点.

(B4.1) $y_{1} y_{3} \in E(G)$. 此时, $G$ 是一个阶数至多为 6 的图. 给 $y_{1}$ 染 $1,\left\{y_{2}, y_{1} y_{3}\right\}$ 染 2 , $\left\{y_{3}, y_{1} y_{4}\right\}$ 染 $3,\left\{y_{1} y_{2}, y_{3} y_{4}\right\}$ 染 $4,\left\{y_{4}, y_{2} y_{3}\right\}$ 染 5 . 若 $d\left(y_{i}\right)=3$, 给 $y_{i} y_{i}^{\prime}$ 染 1 , 给 $y_{i}^{\prime}$ 染 4 , $i=2,4$.

(B4.2) $y_{1} y_{3} \notin E(G)$. 只需考虑 $d\left(y_{2}\right)=d\left(y_{4}\right)=3$ 这种比较复杂的情形. 对 $j \in\{1,3\}$, 令 $y_{j}^{\prime}$ 是除了 $y_{2}$ 和 $y_{4}$ 以外的 $y_{j}$ 的邻点.

(B4.2.1) $y_{1}^{\prime}=y_{3}^{\prime}$. 令 $y_{1}^{\prime \prime} \neq y_{1}, y_{3}$ 是 $y_{1}^{\prime}$ 的第 3 邻点. 图 $G-\left\{y_{1}, y_{2}, y_{3}, y_{4}, y_{2}^{\prime}, y_{4}^{\prime}\right\}$ 有一个 5 -邻点可区别全染色 $\phi$ 应用 $C=\{1,2, \ldots, 5\}$. 假设 $\phi\left(y_{1}^{\prime \prime}\right)=1, \phi\left(y^{\prime} y_{1}^{\prime \prime}\right)=2, \phi\left(y_{1}^{\prime}\right)=3$. 给 $\left\{y_{1}, y_{3}, y_{2}^{\prime}, y_{4}^{\prime}\right\}$ 染 $1,\left\{y_{2} y_{2}^{\prime}, y_{3} y_{4}\right\}$ 染 $2,\left\{y_{1} y_{2}, y_{4} y_{4}^{\prime}\right\}$ 染 $3,\left\{y_{4}, y_{1} y_{1}^{\prime}, y_{2} y_{3}\right\}$ 染 $4,\left\{y_{2}, y_{1} y_{4}, y_{3} y_{1}^{\prime}\right\}$ 染 5 .

(B4.2.2) $y_{1}^{\prime} \neq y_{3}^{\prime}$. 令 $H=G \cup\{x, y\}-\left\{y_{1}, y_{2}, y_{3}, y_{4}, y_{2}^{\prime}, y_{4}^{\prime}\right\}+\left\{y y_{1}^{\prime}, y y_{3}^{\prime}, y x\right\}$, 其中 $x, y$ 是 新增添的顶点. 显然 $H$ 是一个没有 $K_{4}$-图子式的图且 $\Delta(H)=3$ 和 $|V E(H)|<|V E(G)|$. 因此, $H$ 有一个 5 -邻点可区别全染色 $\phi$ 应用 $C=\{1,2, \ldots, 5\}$. 假设 $\phi(y)=1, \phi\left(y y_{1}^{\prime}\right)=2$, $\phi\left(y y_{3}^{\prime}\right)=3, \phi(y x)=4$.

现在给 $\left\{y_{1}, y_{3}\right\}$ 染 $1,\left\{y_{1} y_{1}^{\prime}, y_{2}\right\}$ 染 $2,\left\{y_{3} y_{3}^{\prime}, y_{4}\right\}$ 染 $3,\left\{y_{1} y_{4}, y_{2} y_{3}\right\}$ 染 $4, y_{1} y_{2}$ 染 $a \in\{3,5\}$ 使得 $C_{\phi}\left(y_{1}^{\prime}\right) \neq\{1,2,4, a\}$, 给 $y_{3} y_{4}$ 染 $b \in\{2,5\}$ 使得 $C_{\phi}\left(y_{3}^{\prime}\right) \neq\{1,3,4, b\}$. 最后, 给 $y_{2} y_{2}^{\prime}$ 染 $\{3,5\} \backslash\{a\}$ 中的颜色, 给 $y_{4} y_{4}^{\prime}$ 染 $\{2,5\} \backslash\{b\}$ 中的颜色.

\section{第 2 部分 $\Delta(G) \geqslant 4$.}

由引理 $2, G$ 含有 $(\mathrm{C} 1)-(\mathrm{C} 6)$ 结构之一. 因为 $\Delta(G) \geqslant 4$, 推出 $|C| \geqslant 6$. 如果 $G$ 含有 $(\mathrm{C} 2)$, 则证明与第 1 部分的情形 (B2.1) 相同. 由引理 7 和 8 , 若 $G$ 含有 $(\mathrm{C} 1)$, 则定理为真. 根据引 理 9 , 若 $G$ 含有 (C5), 则定理为真. 如果 $|C| \geqslant 6$, 且 $G$ 含有相邻 2-点, 则类似第 1 部分情 形 (B1) 的证明定理为真. 这隐含着如果 $G$ 含有 $(\mathrm{C} 6)$, 则定理为真. 因此只需要考虑 (C3) 和 (C4) 中没有相邻 2-点的情形.

(C3) 存在一个 3 -圈 $x y z$ 使得 $d(z)=2$ 和 $d(x)=3$.

令 $x^{\prime} \neq y, z$ 是 $x$ 的第 3 个邻点. 图 $G-x z$ 有一个 $(\Delta(G)+2)$ 邻点可区别全染色 $\phi$ 应 用色集 $C=\{1,2, \ldots, \Delta(G)+2\}$. 若 $d(y)=3$, 则证明与第 1 部分的情形 (B3) 相同.

假设 $d(y) \geqslant 4$. 若 $d\left(x^{\prime}\right) \neq 3$, 只需给 $x z$ 正常染色. 假设 $d\left(x^{\prime}\right)=3$. 若 $\phi\left(x^{\prime}\right) \neq \phi(y z)$, 给 $x z$ 染 $C \backslash\left\{\phi(x), \phi(x y), \phi(y z), \phi\left(x x^{\prime}\right), \phi\left(x^{\prime}\right)\right\}$ 中的颜色. 否则, 给 $x$ 染 $C \backslash\left(C_{\phi}\left(x^{\prime}\right) \cup\{\phi(x y)\}\right)$ 中的颜色, 并对 $x z$ 正常染色.

(C4) 存在两个 3 -圈 $x x_{1} z$ 和 $y y_{1} z$ 使得 $d(z)=4$ 和 $d\left(x_{1}\right)=d\left(y_{1}\right)=2$. 
根据前面的情形 (C3), 假设 $d(x), d(y) \geqslant 4$. 令 $H=G-x_{1} z$. 根据归纳假设, $H$ 有一个 $(\Delta(G)+2)$-邻点可区别全染色 $\phi$ 应用 $C=\{1,2, \ldots, \Delta(G)+2\}$. 若 $d(x), d(y) \geqslant 5$, 只需对 $x_{1} z$ 正常染色. 否则, 假设 $d(x)=4$. 令 $C_{\phi}(x)=\{1,2, \ldots, 5\}$. 不失一般性, 又假设 $\phi(x)=1$, $\phi(x z)=4, \phi\left(x x_{1}\right)=5$. 这表明 $6 \notin C_{\phi}(x)$.

(a) $d(y) \geqslant 5$.

若 $6 \notin\left\{\phi(z), \phi\left(y_{1} z\right), \phi(y z)\right\}$, 则给 $z x_{1}$ 染 6 . 否则, 对 $x_{1} z$ 正常染色.

(b) $d(y)=4$. 因为 $\left|C_{\phi}(y)\right|=5$ 且 $|C| \geqslant 6$, 所以存在某种颜色 $a \in C \backslash C_{\phi}(y)$. 如果 $a \geqslant 6$ 且 $a \notin C_{\phi}(z)$, 给 $x_{1} z$ 染 $a$. 如果 $a \geqslant 6$ 且 $a \in C_{\phi}(z)$, 或者 $a \leqslant 5$ 且 $a \notin C_{\phi}(z)$, 给 $x_{1} z$ 正常染 色. 现在假设 $1 \leqslant a \leqslant 5$ 且 $a \in C_{\phi}(z)$. 若 $6 \notin C_{\phi}(z)$, 给 $x_{1} z$ 染 6 . 否则, 对 $x_{1} z$ 进行正常染 色. 定理证毕.

\section{5 下界 $\Delta+1$}

根据 $G$ 的最大度分成两个定理来证明.

定理 5 设 $G$ 是一个 $\Delta(G)=3$ 没有 $K_{4}$-图子式的图. 如果 $G$ 没有相邻 3-点, 则 $\chi_{a}^{\prime \prime}(G)=4$.

证明 $\chi_{a}^{\prime \prime}(G) \geqslant 4$ 显然成立. 对 $|V E(G)|$ 进行归纳证明. 若 $|V E(G)|=7$, 则 $G$ 是 $K_{1,3}$, 易证 $\chi_{a}^{\prime \prime}(G)=4$. 设 $G$ 是一个连通的没有 $K_{4}$-图子式的图, $\Delta(G)=3,|V E(G)| \geqslant 8$, 且不含 相邻 3-点. 由推论 $1, G$ 含有 (A1), (A2), 或 (A3).

断言 1 设 $A=x_{1} x_{2} \cdots x_{k}$ 是 $G$ 的一个 $k$-圈, $3 \leqslant k \leqslant 4, d\left(x_{1}\right)=3, d\left(x_{i}\right)=2$, $i=2,3, \ldots, k$, 则 $\chi_{a}^{\prime \prime}(G)=4$.

证明 设 $x_{1}^{\prime}$ 是 $x_{1}$ 的不在 $A$ 上的邻点. 因为 $G$ 不含相邻 3 -点, $d\left(x_{1}^{\prime}\right) \neq 3$. 令 $H=$ $G-\left\{x_{2}, \ldots, x_{k}\right\}$. 显然, $H$ 不是 3 -圈. 由归纳假设 (或由引理 5 当 $\Delta(H) \leqslant 2$ 时), $H$ 有一个 4 邻点可区别全染色 $\phi$ 应用色集 $C=\{1,2,3,4\}$. 不失一般性, 假设 $\phi\left(x_{1}\right)=1, \phi\left(x_{1} x_{1}^{\prime}\right)=2$. 若 $k=3$, 则给 $x_{2} x_{3}$ 染 $1, x_{2}$ 染 $2, x_{1} x_{3}$ 染 $3,\left\{x_{3}, x_{1} x_{2}\right\}$ 染 4 . 若 $k=4$, 给 $x_{2} x_{3}$ 染 $1,\left\{x_{2}, x_{3} x_{4}\right\}$ 染 $2,\left\{x_{3}, x_{1} x_{4}\right\}$ 染 $3,\left\{x_{4}, x_{1} x_{2}\right\}$ 染 4 . 因此, $\chi_{a}^{\prime \prime}(G)=4$, 断言为真.

(A1) 存在一个叶子 $v$ 相邻于一个顶点 $u$.

令 $H=G-v$. 则 $H$ 是一个连通的没有 $K_{4}$ - 图子式的图, $\Delta(H) \leqslant 3$, 且 $H$ 不含有相 邻 3-点. 显然, $H$ 不是 3 -圈. 由归纳假设或引理 $5, H$ 有一个 4 -邻点可区别全染色 $\phi$ 应用 $C=\{1,2,3,4\}$.

如果 $d(u)=2$, 令 $x \neq v$ 是 $u$ 的另一个邻点. 给 $u v$ 染不同于 $\phi(u), \phi(x), \phi(x u)$ 的颜色, 给 $v$ 染不同于 $\phi(u), \phi(u v)$ 的颜色.

若 $d(u)=3$, 令 $u_{1}, u_{2} \neq v$ 是 $u$ 的另两个邻点. 因为 $G$ 不含相邻 3 -点, $d\left(u_{i}\right) \leqslant 2, i=1,2$. 令 $\phi$ 是 $G-v$ 的 4 -邻点可区别全染色. 只需正常染 $u v$ 和 $v$ 即可.

(A2) 存在一条路 $x_{1} x_{2} x_{3} x_{4}$ 使得 $d\left(x_{2}\right)=d\left(x_{3}\right)=2$.

由断言 1 , 假设 $x_{1} \neq x_{4}$.

(A2.1) $x_{1}$ 和 $x_{4}$ 至少有一个是 2 -点, 假设 $d\left(x_{4}\right)=2$.

令 $x_{5} \neq x_{3}$ 是 $x_{4}$ 的另外一个邻点. 再次由断言 $1, x_{1} \neq x_{5}$, 这表明 $x_{1} x_{4} \notin E(G)$. 由归 纳假设, $G-\left\{x_{2}, x_{3}\right\}+x_{1} x_{4}$ 有一个 4 -邻点可区别全染色 $\phi$ 应用 $C=\{1,2,3,4\}$. 不失一般 性, 假设 $\phi\left(x_{1}\right)=1, \phi\left(x_{1} x_{4}\right)=2, \phi\left(x_{4}\right)=3$. 给 $\left\{x_{1} x_{2}, x_{3} x_{4}\right\}$ 染 $2, x_{2}$ 染 $3, x_{3}$ 染 $1, x_{2} x_{3}$ 染 4 . 
(A2.2) $d\left(x_{1}\right)=d\left(x_{4}\right)=3$.

因为 $G$ 不含相邻 3 -点, 必含有由 $x_{1}$ 出发的另两条路, $P=x_{1} y_{1} \cdots y_{m}$ 和 $Q=x_{1} z_{1} \cdots z_{n}$, 其中 $y_{i}$ 和 $z_{j}$ 都是 2 -点, $1 \leqslant i \leqslant m-1,1 \leqslant j \leqslant n-1$. 由情形 (A2.1), 假设 $2 \leqslant n \leqslant m \leqslant 3$. 由归纳假设, $G-x_{2} x_{3}$ 有一个 4 -邻点可区别全染色 $\phi$ 应用 $C=\{1,2,3,4\}$. 不失一般性, 假 设 $\phi\left(x_{1}\right)=1, \phi\left(x_{1} x_{2}\right)=2$.

断言 2 如果 $\phi\left(x_{4}\right) \neq 2$, 或 $\phi\left(x_{3} x_{4}\right) \neq 1$, 则 $\phi$ 可延拓到 $G$ 上.

证明 令 $\phi\left(x_{3} x_{4}\right)=a, \phi\left(x_{4}\right)=b$. 由颜色 3 和 4 的对称性, 只需考虑以下几种情形.

如果 $a=3$ 且 $b=4$, 给 $x_{2}$ 染 $4, x_{2} x_{3}$ 染 $1, x_{3}$ 染 2 .

如果 $a=1$ 且 $b=3$, 给 $x_{2}$ 染 $4, x_{2} x_{3}$ 染 $3, x_{3}$ 染 2 .

如果 $a=2$ 且 $b \in\{1,3\}$, 或 $a=3$ 且 $b \in\{1,2\}$, 给 $x_{2}$ 染 $3, x_{2} x_{3}$ 染 $1, x_{3}$ 染 4 . 证毕.

由断言 2 , 假设 $\phi\left(x_{4}\right)=2, \phi\left(x_{3} x_{4}\right)=1$. 进一步, 不妨假设 $\phi\left(x_{1} y_{1}\right)=3, \phi\left(x_{1} z_{1}\right)=4$. 现 在考虑以下两种情形.

(A2.2.1) $m=3$. 首先抹掉 $y_{1}, y_{1} y_{2}, y_{2}$ 的颜色. 如果 $\phi\left(y_{2} y_{3}\right) \neq 1$ 或 $\phi\left(y_{3}\right) \neq 2$, 交换 $x_{1} x_{2}$ 和 $x_{1} y_{1}$ 的颜色, 用处理路 $x_{1} x_{2} x_{3} x_{4}$ 的方法来处理路 $x_{1} y_{1} y_{2} y_{3}$. 因此, 结论可由断言 2 推出. 现在假设 $\phi\left(y_{2} y_{3}\right)=1, \phi\left(y_{3}\right)=2$. 显然, $\phi\left(z_{1}\right) \in\{2,3\}$. 如果 $\phi\left(z_{1}\right)=2$, 交换 $x_{1}$ 和 $x_{1} y_{1}$ 的颜 色. 如果 $\phi\left(z_{1}\right)=3$, 交换 $x_{1}$ 和 $x_{1} x_{2}$ 的颜色. 由断言 2 , 以上两种情形都可以延拓到 $G$ 上.

(A2.2.2) $m=n=2$. 如果 $2 \notin\left\{\phi\left(y_{1}\right), \phi\left(y_{1} y_{2}\right)\right\}$, 交换 $x_{1} x_{2}$ 和 $x_{1} y_{1}$ 的颜色, 于是结论由 断言 2 推出. 现在假设 $2 \in\left\{\phi\left(y_{1}\right), \phi\left(y_{1} y_{2}\right)\right\}$, 类似地 $2 \in\left\{\phi\left(z_{1}\right), \phi\left(z_{1} z_{2}\right)\right\}$. 应用断言 2 , 我们分 别处理下面三种情形:

(A2.2.2a) $\phi\left(y_{1}\right)=\phi\left(z_{1}\right)=2$. 如果 $\phi\left(y_{1} y_{2}\right) \neq 1$, 交换 $x_{1}$ 和 $x_{1} y_{1}$ 的颜色. 如果 $\phi\left(y_{1} y_{2}\right)=1$, 类似地 $\phi\left(z_{1} z_{2}\right)=1$, 先给 $y_{1}$ 改染 $\{3,4\} \backslash\left\{\phi\left(y_{2}\right)\right\}$ 中的颜色, 然后交换 $x_{1} x_{2}$ 和 $x_{1} y_{1}$ 的颜色.

(A2.2.2b) $\phi\left(y_{1}\right)=\phi\left(z_{1} z_{2}\right)=2$. 注意到, $\phi\left(z_{1}\right)=3$. 交换 $x_{1}$ 和 $x_{1} z_{1}$ 的颜色. 如果 $\phi\left(y_{1} y_{2}\right)=\phi\left(z_{1}\right)=2$, 我们归结为上面情形 (A2.2.2a) 的讨论.

(A2.2.2c) $\phi\left(y_{1} y_{2}\right)=\phi\left(z_{1} z_{2}\right)=2$. 容易看出 $\phi\left(y_{1}\right)=4, \phi\left(z_{1}\right)=3$. 只需交换 $x_{1}$ 和 $x_{1} x_{2}$ 的颜色.

(A3) 存在一个 4 -圈 $y_{1} y_{2} y_{3} y_{4}$ 使得 $d\left(y_{1}\right)=d\left(y_{3}\right)=3$ 和 $d\left(y_{2}\right)=d\left(y_{4}\right)=2$.

对 $i=1,3$, 令 $y_{i}^{\prime}$ 是 $y_{i}$ 的不同于 $y_{2}$ 和 $y_{4}$ 的邻点. 于是, $d\left(y_{i}^{\prime}\right)=2$. 令 $y_{i}^{\prime \prime} \neq y_{i}$ 是 $y_{i}^{\prime}$ 的另 一个邻点. 由 (A2), 可以假设 $y_{1}^{\prime \prime}$ 和 $y_{3}^{\prime \prime}$ 都是 3 -点.

如果 $y_{1}^{\prime}=y_{3}^{\prime}$, 则 $G$ 是一个阶为 5 的图, 结论显然成立. 假设 $y_{1}^{\prime} \neq y_{3}^{\prime}$, 又由 (A2) 得 $y_{1}^{\prime} y_{3}^{\prime} \notin E(G)$. 考虑图 $H=G-\left\{y_{1}, y_{2}, y_{3}, y_{4}\right\}+y_{1}^{\prime} y_{3}^{\prime}$. 显然, $H$ 是一个没有 $K_{4}$-图子式的 图, $\Delta(H) \leqslant 3$, 且 $H$ 不含相邻 3 -点. 由归纳假设, $H$ 有一个 4 -邻点可区别全染色 $\phi$ 应用 $C=\{1,2,3,4\}$. 不失一般性, 假设 $\phi\left(y_{1}^{\prime}\right)=1, \phi\left(y_{1}^{\prime} y_{3}^{\prime}\right)=2, \phi\left(y_{3}^{\prime}\right)=3$. 在 $G$ 中, 给 $\left\{y_{3}, y_{1} y_{2}\right\}$ 染 $1,\left\{y_{2}, y_{4}, y_{1} y_{1}^{\prime}, y_{3} y_{3}^{\prime}\right\}$ 染 $2,\left\{y_{1}, y_{3} y_{4}\right\}$ 染 $3,\left\{y_{1} y_{4}, y_{2} y_{3}\right\}$ 染 4 . 证明完毕.

定理 6 设 $G$ 是一个 $\Delta(G) \geqslant 4$ 没有 $K_{4}$-图子式的图. 如果 $G$ 没有相邻的最大度点, 则 $\chi_{a}^{\prime \prime}(G)=\Delta(G)+1$.

证明 显然, $\chi_{a}^{\prime \prime}(G) \geqslant \Delta(G)+1$. 为了证明 $\chi_{a}^{\prime \prime}(G) \leqslant \Delta(G)+1$, 对 $|V E(G)|$ 进行归 纳. 如果 $|V E(G)| \leqslant 5$, 那么结论显然成立. 假设 $G$ 是一个连通的没有 $K_{4}$-图子式的图使得 $\Delta(G) \geqslant 4,|V E(G)| \geqslant 6$, 且 $G$ 不含相邻的最大度点. 
首先, 假设 $\Delta(G) \geqslant 5$. 由引理 $2, G$ 含有 $(\mathrm{C} 1)-(\mathrm{C} 6)$ 之一. 注意到, 现在至少有 6 种颜 色可以利用. 如果 $G$ 含有 (C2) 或 (C6), 证明过程与定理 4 中情形 (B2.1) 相同. 如果 $G$ 含 有 (C3), 证明过程与定理 4 中情形 (B3) 相同. 如果 $G$ 含有 (C4), 证明过程与定理 4 中情 形 (B4) 相同. 如果 $G$ 含有 (C5), 结论可以由引理 9 推出. 假设 $G$ 含有 $(\mathrm{C} 1)$, 即一个 $k$-点, $k \geqslant 3$, 与一个叶子和至少 $k-2$ 个 $2^{-}$-点相邻. 如果 $k=3$, 结论由引理 7 推出. 如果 $k \geqslant 4$, 结论由引理 8 推出.

现在假设 $\Delta(G)=4$. 于是, $|C|=5$. 由引理 $4, G$ 含有 (B'1)、(B2)、(B3) 和 (B4) 之一. 如果 $G$ 含有 (B2), 则证明过程与定理 4 中情形 (B2) 相同.

$\left(\mathrm{B}^{\prime} 1\right)$ 存在一个叶子 $v$ 相邻于一个顶点 $u$ 使得 $d(u) \neq 3$, 或者 $d(u)=3$ 且 $n_{3}(u) \leqslant 1$.

如果 $d(u) \leqslant 2$, 证明过程与定理 4 中情形 (B1) 相同. 如果 $d(u)=4$, 则 $u$ 的邻点不可能 是 4-点. 图 $G-u v$ 的任意 5 -邻点可区别全染色均可延拓到 $G$ 上. 如果 $d(u)=3$ 且 $n_{3}(u) \leqslant 1$, 结论可由引理 7 推出.

(B3) 存在一个 3 -圈 $x y z$ 使得 $d(x)=3$, 或者 $d(z)=2$, 或者 $d(z)=3$ 且 $z$ 与一个叶子 $z^{\prime}$ 相邻.

令 $x^{\prime} \neq z, y$ 是 $x$ 的另一个邻点. 由 (B2), 假设 $d(y) \geqslant 3$. 如果 $z^{\prime}$ 存在, 则 $y$ 和 $x$ 都必是 3 -点, 因为否则 $\left(\mathrm{B}^{\prime} 1\right)$ 出现

(B3.1) $d(y)=4$ 且 $d(z)=2$.

由归纳假设, $G-x z$ 有一个 5 -邻点可区别全染色 $\phi$ 应用 $C=\{1,2, \ldots, 5\}$. 如果 $d\left(x^{\prime}\right) \neq 3$, 只需对 $x z$ 正常染色. 假设 $d\left(x^{\prime}\right)=3, C_{\phi}\left(x^{\prime}\right)=\{1,2,3,4\}$. 如果 $5 \notin\{\phi(x), \phi(x y), \phi(y z)\}$, 给 $x z$ 染 5. 如果 $5 \in\{\phi(x), \phi(x y)\}$, 给 $x z$ 正常染色. 如果 $5 \notin\{\phi(x), \phi(x y)\}$ 且 $\phi(y z)=5$, 我们 交换 $y z$ 和 $x y$ 的颜色, 给 $x z$ 正常染色即可.

(B3.2) $d(y)=3$ 且 $z^{\prime}$ 存在.

令 $y^{\prime} \neq x, z$ 是 $y$ 的另一个邻点. 图 $G-\{z, x y\}$ 有一个 5 -邻点可区别全染色 $\phi$ 应 用 $C=\{1,2, \ldots, 5\}$. 如果 $d\left(x^{\prime}\right)=d\left(y^{\prime}\right)=3$, 证明过程与定理 4 中情形 (B3) 相同. 假设 $d\left(y^{\prime}\right) \neq 3, \phi\left(x^{\prime}\right)=1, \phi\left(x x^{\prime}\right)=2, \phi\left(y^{\prime}\right)=a, \phi\left(y y^{\prime}\right)=b$. 此外, 若 $d\left(x^{\prime}\right)=3$, 我们进一步假设 $C_{\phi}\left(x^{\prime}\right)=\{1,2,3,4\}$. 根据颜色 3 和 4 的对称性, 证明分成以下 4 种情形.

(B3.2.1) $a=1$.

如果 $b=2$, 给 $x z$ 染 $1,\left\{y, z z^{\prime}\right\}$ 染 $3,\{x, y z\}$ 染 $4,\{x y, z\}$ 染 5 .

如果 $b=3$, 给 $y$ 染 2 , 其余元素的染色与上面情形相同.

如果 $b=5$, 给 $y z$ 染 $1,\left\{y, z z^{\prime}\right\}$ 染 $2,\{z, x y\}$ 染 $3, x z$ 染 $4, x$ 染 5 .

(B3.2.2) $a=2$.

如果 $b=1$, 给 $z$ 染 $1, z z^{\prime}$ 染 $2,\{y, x z\}$ 染 $3,\{x, y z\}$ 染 $4, x y$ 染 5 .

如果 $b=3$, 给 $\{y, x z\}$ 染 $1, z$ 染 $2, z z^{\prime}$ 染 $3,\{x, y z\}$ 染 $4, x y$ 染 5 .

如果 $b=5$, 给 $\{z, x y\}$ 染 $1, y z$ 染 $2, x z$ 染 $3,\left\{y, z z^{\prime}\right\}$ 染 $4, y$ 染 5 .

(B3.2.3) $a=3$.

如果 $b=1$, 给 $z z^{\prime}$ 染 $1, y$ 染 $2, x z$ 染 $3,\{x, y z\}$ 染 $4,\{z, x y\}$ 染 5 .

如果 $b=2$, 给 $y$ 染 1 , 其余元素的染色与上面情形相同.

如果 $b=4$, 给 $\left\{y, z z^{\prime}\right\}$ 染 $1, y z$ 染 $2, x z$ 染 $3, x$ 染 $4,\{z, x y\}$ 染 5 .

如果 $b=5$, 给 $\{z, x y\}$ 染 $1, z z^{\prime}$ 染 $2, y z$ 染 $3,\{y, x z\}$ 染 $4, y$ 染 5 . 
(B3.2.4) $a=5$.

如果 $b=1$, 给 $z$ 染 $1, y$ 染 $2,\left\{x y, z z^{\prime}\right\}$ 染 $3,\{x, y z\}$ 染 $4, x z$ 染 5 .

如果 $b=2$, 给 $y z$ 染 $1, z$ 染 $2, x y$ 染 $3,\{y, x z\}$ 染 $4,\left\{x, z z^{\prime}\right\}$ 染 5 .

如果 $b=3$, 给 $x y$ 染 $1, z$ 染 $2, z z^{\prime}$ 染 $3,\{y, x z\}$ 染 $4,\{x, y z\}$ 染 5 .

(B4) 存在一个 4 -圈 $y_{1} y_{2} y_{3} y_{4}$ 使得 $y_{2}$ 和 $y_{4}$ 或是 2 -点或是可控制的 3 -点.

对 $i \in\{2,4\}$, 如果 $d\left(y_{i}\right)=3$, 令 $y_{i}^{\prime}$ 是与 $y_{i}$ 相邻的叶子. 不妨设 $y_{1}$ 和 $y_{3}$ 都是 $3^{+}$-点, 否 则 (B2) 将出现. 如果 $y_{1}$ 和 $y_{3}$ 至少之一是 4-点, 比如 $d\left(y_{1}\right)=4$, 则 $d\left(y_{2}\right)=d\left(y_{4}\right)=2$, 否则 $\left(\mathrm{B}^{\prime} 1\right)$ 将出现. 图 $G-\left\{y_{2}, y_{4}\right\}+y_{1} y_{3}$ 有一个 5 -邻点可区别全染色 $\phi$ 应用 $C=\{1,2, \ldots, 5\}$. 假设 $\phi\left(y_{1}\right)=1, \phi\left(y_{1} y_{3}\right)=2, \phi\left(y_{3}\right)=3$. 现在先给 $\left\{y_{1} y_{2}, y_{3} y_{4}\right\}$ 染 2 , 然后染 $y_{1} y_{4}$ 和 $y_{2} y_{3}$ 使 得 $y_{3}$ 和 $y_{3}^{\prime}$ 之间不存在冲突, 其中 $y_{3}^{\prime}$ 是 $y_{3}$ 的不同于 $y_{2}$ 和 $y_{4}$ 的邻点.

假设 $d\left(y_{1}\right)=d\left(y_{3}\right)=3$. 如果 $y_{1} y_{3} \in E(G)$, 则 $G$ 中含有 (B3). 如果 $y_{1} y_{3} \notin E(G)$, 证明 与定理 4 中情形 (B4) 相同. 证毕.

容易看到, 第 1 节叙述的主要定理可由引理 6, 定理 4-6 推导出来.

\section{参考文献}

1 Zhang Z, Liu L, Wang J. Adjacent strong edge coloring of graphs. Appl Math Lett, 15: 623-626 (2002)

2 Balister P N, Győri E, Lehel J, et al. Adjacent vertex distinguishing edge-colorings. SIAM J Discrete Math, 21: 237-250 (2007)

3 Hatami H. $\Delta+300$ is a bound on the adjacent vertex distinguishing edge chromatic number. J Combin Theory Ser B, 95: 246-256 (2005)

4 Behzad M. Graphs and their chromatic numbers. PhD Thesis. Michigan: Michigan State University, 1965

5 Vizing V. Some unsolved problems in graph theory (in Russian). Uspekhi Mat Nauk, 23: 117-134 (1968)

6 张忠辅, 陈祥恩, 李敬文, 等. 关于图的邻点可区别全染色. 中国科学 A, 34(5): 574-583 (2004)

7 Chen $\mathrm{X}$. On the adjacent vertex distinguishing total coloring numbers of graphs with $\Delta=3$. Discrete Math, 308: 4003-4007 (2008)

8 Wang $\mathrm{H}$. On the adjacent vertex distinguishing total chromatic number of the graphs with $\Delta(G)=3 . J$ Combin Optim, 14: 87-109 (2007)

9 Wang Y, Wang W. Adjacent vertex distinguishing total colorings of outerplanar graphs. J Combin Optim, to appear

10 Lih K-W, Wang W, Zhu X. Coloring the square of a $K_{4}$-minor free graph. Discrete Math, 269: 303-309 (2003) 